\title{
Not your usual tRNA synthetase: hWARS serves as an enterovirus entry factor
}

\author{
Stanley Perlman ${ }^{1,2}$ and Tom Gallagher ${ }^{3}$ \\ 'Department of Microbiology and Immunology, University of lowa and 'State Key Laboratory of Respiratory Disease, National Clinical Research Center for Respiratory Disease, Guangzhou Institute of \\ Respiratory Health, the First Affiliated Hospital of Guangzhou Medical University, Guangzhou, China. ${ }^{3}$ Department of Microbiology and Immunology, Loyola University, Chicago, Illinois, USA.
}

\begin{abstract}
Enteroviruses, including subtype EV-A71, infect the brain, liver, heart, and other organs, causing a myriad of human diseases. This spectrum of disease is thought to be due, in part, to differential binding to host cells, and additional knowledge of enterovirus cell entry is essential for therapeutic development. In this issue of the $J C l$, Yeung et al. provide evidence of a novel EV-A71 entry factor, a host-produced tryptophan tRNA synthetase (hWARS), that facilitates entry of multiple subtypes of enteroviruses. hWARS is a cytoplasmic enzyme that is essential for translation but also upregulated and secreted during inflammatory processes. The results of this study support the notion of secreted hWARS as an unconventional virus entry factor that raises interesting questions about mechanisms by which inflammation and a tRNA synthetase facilitate viral pathogenesis.
\end{abstract}

\section{Enteroviruses: one genus,} a wide range of diseases

Enteroviruses encompass a large number of human pathogens, including ones that cause myocarditis, aseptic meningitis, and encephalitis (1). Probably the best-known enteroviruses are the polioviruses, the causative agent of poliomyelitis. Infection with poliovirus is largely controlled by vaccination, although some strains of WT polio and vaccine-derived virus continue to circulate and cause disease (2). However, even with the nearly complete eradication of poliovirus, the syndrome of poliomyelitis and associated long-term neurological disability remain an important medical problem. Enterovirus $\mathrm{A} 71$ (EV-A71) is foremost among these agents that cause encephalitis and acute flaccid paralysis (3). EV-A71 is also one of the etiological agents of hand, foot, and mouth disease, a common but non-life-threatening illness. For reasons that are not clear, EV-A71 infection is most prevalent in East Asia and along the Pacific rim and, like poliovirus, primarily infects toddlers and young children.
Two vaccines for EV-A71 are licensed for use in China, and others are under development and in clinical trials (4). No therapy is currently available to treat the infection.

The rational development of antiviral therapies will require a further understanding of enterovirus pathogenesis. Among the unclear features are the first steps of enterovirus infection, when viruses bind receptors on susceptible host cells. For EV-A71, P-selectin glycoprotein ligand 1 (PSGL1) (5) and the scavenger receptor class $\mathrm{B}$ member 2 (SCARB2) (6) are known receptors. However, PSGL1 is not expressed on several enterovirus-susceptible cell types, and both PSGL1 and SCARB2 are recognized by only a small subset of serotype A enteroviruses, making it likely that additional enterovirus cell entry factors are at play.

\section{An unexpected and unconventional entry factor} In this issue, Yeung et al. report the discovery of an additional enterovirus cell entry factor. Using a lentiviral shRNA library

Related Article: p. 5163

screen, as well as specific knockdown shRNAs in EV-A71-sensitive cells, the authors identified human tryptophanyltRNA synthetase (hWARS) as an EV-A71 susceptibility determinant (7). A definitive role for hWARS in EV-A71 entry was further demonstrated by the ability of induced hWARS expression to sensitize EV-A71resistant cells to infection. Recombinant hWARS bound directly to EV-A71 particles in coimmunoprecipitation tests, and hWARS expression in mice via lentivirus transduction increased EV-A71 susceptibility, demonstrating that expression of this molecule by itself increases infection and virus-induced neurological disease. These findings all point to hWARS as a receptor for the virus; however, Yeung et al. are careful to call hWARS an entry factor until its role in facilitating infection susceptibility is further unraveled (see below).

Knockdown of hWARS also variably diminished susceptibility of cells to other enteroviruses, including EV-D68, coxsackie A viruses, and others, suggesting that this protein also has a broad role in cell entry. EV-D68 is a recently identified causative agent of recent outbreaks of upper and lower tract respiratory disease that primarily infects pediatric patients throughout North America, Asia, and Europe and results in hospitalization in many cases (8). EV-D68 and other enteroviruses appear unable to enter cells via PSGL1 or SCARB2 (9), lending credence to hWARS as a more relevant, broadly acting enterovirus entry factor. However, future studies should focus on determining the precise role of all three factors in EV-A71 entry and whether the functionality of these factors is overlapping or complementary and should provide a further understanding of enterovirus pathogenesis.

On the surface, the most curious and unexpected aspect of hWARS as an enterovirus receptor is that tRNA synthetases are expected to be cytoplasmic proteins, with well-defined functions in protein syn- 


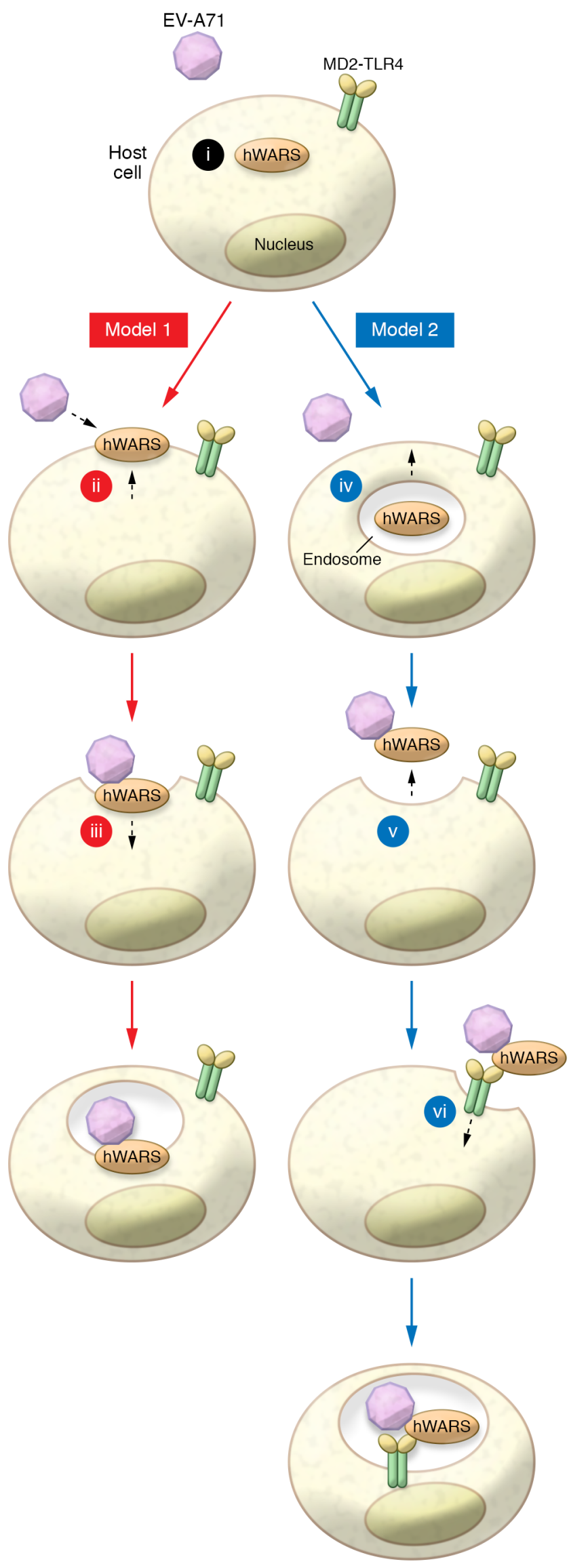

Figure 1. Possible mechanisms to explain surface expression of a tRNA synthetase. hWARS is typically expressed as a cytoplasmic protein (i); however, in this issue, Yeung et al. demonstrate that hWARS is secreted in response to IFN- $\gamma$ stimulation, and the secreted form binds to and promotes entry of EV-A71. In Model 1, hWARS is directly transported and anchored to the plasma membrane by an unknown mechanism (ii). EV-A71 then enters cells by binding to hWARS on the surface (iii). In Model 2, hWARS is enclosed in a vesicle in the cell (iv) and is released into the extracellular medium, probably after fusion of the vesicle with the cell membrane (v). Free hWARS (iv) then binds to virus, with the complex entering cells via binding to MD2-TLR4 or another surface membrane-bound protein. At this point, the entire EV-A71-hWARS-MD2-TLR4 enters the cell via endocytosis, prior to RNA delivery into the cytoplasm.

thesis. However, hWARS is a remarkably multifunctional protein, and several studies have demonstrated that it has additional roles as an extracellular ligand. For example, an N-terminal truncated form of hWARS has angiostatic properties (10). This activity depends on secreted hWARS and its binding to E-cadherin (11). A fulllength form of hWARS was shown to have anti-infective properties (10), and this activity also requires extracellular hWARS, which increases after pathogen exposure and binds to MD2-TLR4, thereby activating the innate immune response (12). More recently, hWARS was shown to mediate high-affinity tryptophan uptake, either directly or indirectly (13). Tryptophan is the precursor to kynurenine, which has multiple physiological effects, including activation of the aryl hydrocarbon receptor, that mediate inflammation (14).

Thus, while hWARS meets the requirement that a virus receptor be displayed extracellularly on the plasma membrane, it does not fit with classical virus receptors, which are firmly anchored in membranes in susceptible cells. One possibility is that freely diffusing, secreted hWARS binds extracellular EV-A71 particles, with the EV-A71-hWARS complex then binding to a surface protein that in turn facilitates virus entry into host cells. In this context, hWARS might serve as an entry adaptor with an as-yet unidentified surface protein (possibly E-cadherin or MD2-TLR4) that functions as the enterovirus receptor (Figure 1). There is precedence for adaptors in viral infection; for example, the bivalent growth 
arrest-specific 6 (GAS6) protein binds to lipids on enveloped viruses and transmembrane receptors on host cells, thereby forming an essential connecting bridge during virus entry (15). Likewise, antibody-dependent enhancement of virus infection, most notably dengue virus, involves an adaptor molecule, in this case, an antibody, which serves as the bridging agent that mediates entry into host cells (16).

An additional feature of hWARS, with relevance to viral infection, is that it is the only tRNA synthetase that is IFN- $\gamma$ induced (17). Yeung et al. demonstrated that IFN- $\gamma$ not only increases hWARS expression but also results in increased membrane localization and increased susceptibility to EV-A71 infection. EV-A71, as well as other enteroviruses, will induce an inflammatory response, including increased expression of IFN $-\gamma$; therefore, in this setting, IFN- $\gamma$ can be considered a proviral factor that positively reinforces an EV-A71/IFN- $\gamma /$ hWARS loop. Previously, IFN- $\gamma$ was shown to augment cell entry of the human respiratory coronavirus $\mathrm{HCoV}-\mathrm{OC} 43$ by increasing expression of IFN-induced transmembrane proteins (IFITMs), which have well-described antiviral properties (18). Hence, in both EV-A71 and $\mathrm{HCoV}-\mathrm{OC} 43$ infections, viruses hijack molecules that are normally antiviral to enhance virus replication. These results also raise the possibility that some of the variability in cellular tropism and clinical disease observed in EV-A71-infected patients results from differences in IFN- $\gamma$ levels.

\section{Concluding remarks}

The intriguing results of Yeung et al. raise several points for future consideration. With appreciation for the significant functions of extracellular aminoacyl tRNA synthetases, there will be greater attention paid to how these proteins are secreted. These proteins lack N-terminal ER signal peptides, and thus their secretion follows unclear and unconventional pathways, perhaps akin to other secreted components of the innate immune system such as IL-1 $\beta$ (19). With the new understanding that a tRNA synthetase functions in virus attachment, it will be important to obtain structural data showing how hWARS interacts molecularly with EV-A71 and cellular transmembrane proteins. Recent structural advances using cryoelectron microscopy (20) make it feasible to explore such interactions and should provide additional information about the interaction of the virus with hWARS and the other putative receptors SCARB2 and PSGL1. It will also be of interest to determine whether the tRNA synthetase enzymatic activity of hWARS is required for enterovirus entry and whether angiostatic, immunomodulatory, and proviral activities depend on distinct structural elements of hWARS and its orthologs in experimental animal models. hWARS is expressed as both a full-length protein and a shorter, alternatively spliced variant. Whether one or both forms of hWARS facilitate EV-A71 entry or that of other enteroviruses will be important to determine. This will be especially true in the context of the infection of neurons, particularly anterior horn cells, as infection of these cells results in acute flaccid paralysis and long-term neurological disability, the most feared complications of EV-A71 infection (21).

\section{Acknowledgments}

SP and TG are supported by grants from the NIH (PO1 AI060699, to SP and TG, and RO1 NS36592, to SP). We thank Josalyn Cho (University of Iowa) for helping with the figure.

Address correspondence to: Stanley Perlman, Department of Microbiology and Immunology, University of Iowa, Iowa City, Iowa 52242, USA. Phone: 319.335.8549; Email: stanley-perlman@uiowa.edu.

1. Baggen J, Thibaut HJ, Strating JRPM, van Kuppeveld FJM. The life cycle of non-polio enteroviruses and how to target it. Nat Rev Microbiol. 2018;16(6):368-381.

2. Blake IM, et al. Type 2 poliovirus detection after global withdrawal of trivalent oral vaccine. N Engl J Med. 2018;379(9):834-845.

3. Bitnun A, Yeh EA. Acute flaccid paralysis and enteroviral infections. Curr Infect Dis Rep. 2018;20(9):34 .

4. Mao Q, Wang Y, Bian L, Xu M, Liang Z. EV-A71 vaccine licensure: a first step for multivalent enterovirus vaccine to control HFMD and other severe diseases. Emerg Microbes Infect. 2016;5(7):e75.

5. Nishimura Y, Shimojima M, Tano Y, Miyamura T, Wakita T, Shimizu H. Human P-selectin glycoprotein ligand-1 is a functional receptor for enterovirus 71. Nat Med. 2009;15(7):794-797.

6. Yamayoshi S, et al. Scavenger receptor B2 is a cellular receptor for enterovirus 71. Nat Med. 2009;15(7):798-801.

7. Yeung ML, et al. Human tryptophanyl-tRNA synthetase is an IFN- $\gamma$-inducible entry factor for Enterovirus. JClin Invest. 2018;128(11):5163-5177.

8. Holm-Hansen CC, Midgley SE, Fischer TK. Global emergence of enterovirus D68: a systematic review. Lancet Infect Dis. 2016;16(5):e64-e75.

9. Yamayoshi S, et al. Human SCARB2-dependent infection by coxsackievirus A7, A14, and A16 and enterovirus 71. J Virol. 2012;86(10):5686-5696.

10. Wakasugi K, et al. A human aminoacyl-tRNA synthetase as a regulator of angiogenesis. Proc Natl Acad Sci U S A. 2002;99(1):173-177.

11. Tzima E, Reader JS, Irani-Tehrani M, Ewalt KL, Schwartz MA, Schimmel P. VE-cadherin links tRNA synthetase cytokine to anti-angiogenic function. J Biol Chem. 2005;280(4):2405-2408.

12. Ahn YH, et al. Secreted tryptophanyl-tRNA synthetase as a primary defence system against infection. Nat Microbiol. 2016;2:16191.

13. Miyanokoshi M, Yokosawa T, Wakasugi K. Tryptophanyl-tRNA synthetase mediates high-affinity tryptophan uptake into human cells. J Biol Chem. 2018;293(22):8428-8438.

14. Stockinger B, Di Meglio P, Gialitakis M, Duarte $\mathrm{JH}$. The aryl hydrocarbon receptor: multitasking in the immune system. Annu Rev Immunol. 2014;32:403-432.

15. Morizono K, et al. The soluble serum protein Gas6 bridges virion envelope phosphatidylserine to the TAM receptor tyrosine kinase Axl to mediate viral entry. Cell Host Microbe. 2011;9(4):286-298.

16. Katzelnick LC, et al. Antibody-dependent enhancement of severe dengue disease in humans. Science. 2017;358(6365):929-932.

17. Bhutia YD, Babu E, Ganapathy V. Interferon- $\gamma$ induces a tryptophan-selective amino acid transporter in human colonic epithelial cells and mouse dendritic cells. Biochim Biophys Acta. 2015;1848(2):453-462.

18. Zhao X, et al. Interferon induction of IFITM proteins promotes infection by human coronavirus OC43. Proc Natl Acad Sci U S A. 2014;111(18):6756-6761.

19. Monteleone M, Stow JL, Schroder K. Mechanisms of unconventional secretion of IL-1 family cytokines. Cytokine. 2015;74(2):213-218.

20. Cheng Y. Single-particle cryo-EM-How did it get here and where will it go. Science. 2018;361(6405):876-880.

21. Xing J, et al. Pathologic studies of fatal encephalomyelitis in children caused by enterovirus 71 . Am J Clin Pathol. 2016;146(1):95-106. 